\title{
Bright light therapy for bipolar depression: A review of 6 studies
}

Sy Atezaz Saeed, MD, MS, and Radhika J. Kothadia, MD

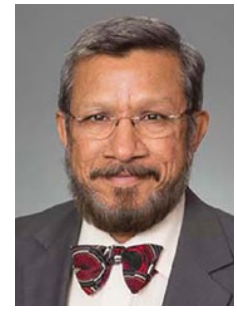

Sy Atezaz Saeed, MD, MS Department Editor

\section{Recent evidence} suggests BLT may help reduce depressive symptoms

\section{f}

Discuss this article at www.facebook.com/ MDedgePsychiatry

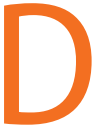
epressive episodes are part of DSM-5 criteria for bipolar II disorder, and are also often experienced by patients with bipolar I disorder. ${ }^{1}$ Depressive episodes predominate the clinical course of bipolar disorder. ${ }^{2,3}$ Compared with manic and hypomanic episodes, bipolar depressive episodes have a stronger association with long-term morbidity, suicidal behavior, and impaired functioning. ${ }^{4,5}$ Approximately $20 \%$ to $60 \%$ of patients with bipolar disorder attempt suicide at least once in their lifetime, and $4 \%$ to $19 \%$ die by suicide. Compared with the general population, the risk of death by suicide is 10 to 30 times higher in patients with bipolar disorder. ${ }^{6}$

Treatment of bipolar depression is less investigated than treatment of unipolar depression or bipolar mania. The mainstays of treatment for bipolar depression include mood stabilizers (eg, lithium, valproic acid, or lamotrigine), second-generation antipsychotics (eg, risperidone, quetiapine, lurasidone, or olanzapine), adjunctive antidepressants (eg, selective serotonin reuptake inhibitors or bupropion), and combinations of the above. While significant progress has been made in the treatment of mania, achieving remission for patients with bipolar depression remains a challenge. Anti-manic medications reduce depressive symptoms in only one-third of patients. $^{7}$ Antidepressant monotherapy can induce hypomania and rapid cycling. ${ }^{8}$ Electroconvulsive therapy has also been used for treatment-resistant bipolar depression, but is usually reserved as a last resort. ${ }^{9}$
Research to investigate novel therapeutics for bipolar depression is a high priority. Patients with bipolar disorder are susceptible to environmental cues that alter circadian rhythms and trigger relapse. Recent studies have suggested that bright light therapy (BLT), an accepted treatment for seasonal depression, also may be useful for treating nonseasonal depression..$^{10}$ Patients with bipolar depression frequently have delayed sleep phase and atypical depressive features (hypersomnia, hyperphagia, and lethargy), which predict response to light therapy. ${ }^{11}$ In this article, we review 6 recent studies that evaluated the efficacy and safety of BLT for treating bipolar depression (Table, ${ }^{12-17}$ page 41).

\section{Wang S, Zhang Z, Yao L, et al. Bright light therapy in treatment of patients with bipolar disorder: a systematic review and meta-anal- ysis. PLoS ONE. 2020;15(5):e0232798. doi: 10.1371/journal.pone.0232798}

In this meta-analysis, Wang et $\mathrm{a}^{12}$ examined the role of BLT in treating bipolar depression. They also explored variables of BLT, including duration, timing, color, and color

Dr. Saeed is Professor and Chair, Department of Psychiatry and Behavioral Medicine, East Carolina University Brody School of Medicine, Greenville, North Carolina. Dr. Saeed is CURRENT PsYCHIATRY's Department Editor, Psychiatry Journal Club. Dr. Kothadia is a PGY-1 Psychiatry Resident, Prisma Health-Midlands, Columbia, South Carolina.

Disclosures

The authors report no financial relationships with any companies whose products are mentioned in this article, or with manufacturers of competing products.

doi: $10.12788 /$ cp.0114 


\section{Bright light therapy for bipolar depression: 6 studies}

\begin{tabular}{|c|c|c|}
\hline Study & Design & Outcomes \\
\hline Wang et $\mathrm{al}^{12}$ & $\begin{array}{l}\text { Review and meta-analysis of } 5 \text { RCTs and } \\
7 \text { cohort studies evaluating BLT for } 847 \\
\text { patients with bipolar depression }\end{array}$ & $\begin{array}{l}\text { Compared with placebo, BLT } \\
\text { significantly decreased depression } \\
\text { severity }(P<.05)\end{array}$ \\
\hline Lam et $\mathrm{al}^{13}$ & $\begin{array}{l}\text { Review and meta-analysis of } 7 \text { RCTs } \\
\text { evaluating BLT for } 259 \text { patients with } \\
\text { bipolar depression }\end{array}$ & $\begin{array}{l}\text { Compared with control conditions, BLT } \\
\text { was associated with a small to moderate } \\
\text { but statistically significant improvement } \\
\text { in clinician-rated depressive symptoms } \\
(P=.03)\end{array}$ \\
\hline Hirakawa et al $^{14}$ & $\begin{array}{l}\text { Review and meta-analysis of } 4 \text { RCTs } \\
\text { evaluating BLT for } 190 \text { patients with } \\
\text { bipolar depression }\end{array}$ & $\begin{array}{l}\text { BLT had a significant effect on response } \\
\text { rates }(P=.002) \text { but not on remission } \\
\text { rates }(P=.34)\end{array}$ \\
\hline $\begin{array}{l}\text { D'Agostino } \\
\text { et al }{ }^{15}\end{array}$ & $\begin{array}{l}\text { Review of } 6 \text { studies evaluating triple } \\
\text { chronotherapy (total sleep deprivation, } \\
\text { sleep phase advance, and BLT) for } 190 \\
\text { patients with MDD or bipolar depression }\end{array}$ & $\begin{array}{l}\text { All studies reported improvement in } \\
\text { HAM-D scores at the end of treatment, } \\
\text { with response rates ranging from } 50 \% \\
\text { to } 84 \%\end{array}$ \\
\hline $\begin{array}{l}\text { Dallaspezia } \\
\text { and Benedetti16 }\end{array}$ & $\begin{array}{l}\text { Meta-analysis of } 11 \text { studies (including } \\
5 \text { RCTs) evaluating BLT for depressive } \\
\text { symptoms in } 195 \text { patients }\end{array}$ & $\begin{array}{l}\text { Analyses of all } 11 \text { studies and of just } \\
\text { the } 5 \text { RCTs found BLT had a positive } \\
\text { significant effect on depressive } \\
\text { symptoms (both } P<.001 \text { ) }\end{array}$ \\
\hline $\begin{array}{l}\text { Takeshima } \\
\text { et } \mathrm{al}^{17}\end{array}$ & $\begin{array}{l}\text { Review and meta-analysis of } 6 \text { studies } \\
\text { evaluating BLT for manic and depressive } \\
\text { symptoms in } 280 \text { patients with bipolar } \\
\text { disorder }\end{array}$ & $\begin{array}{l}\text { No significant differences were found } \\
\text { between BLT and placebo for rate of } \\
\text { remission from depressive episodes, } \\
\text { rate of manic switching, or depressive } \\
\text { symptom scores }\end{array}$ \\
\hline
\end{tabular}

temperature, and how these factors may affect the severity of depressive symptoms.

\section{Study design}

- Two researchers conducted a systematic literature search on PubMed, Web of Science, Embase, Cochrane Library, and Cumulative Index of Nursing and Allied Health Literature (CINAHL), as well as 4 Chinese databases from inception to March 2020. Search terms included "phototherapy," "bright light therapy," "bipolar disorder," and "bipolar affective disorder."

- Inclusion criteria called for randomized controlled trials (RCTs) or cohort studies that used a clearly defined diagnosis of bipolar depression. Five RCTs and 7 cohort studies with a total of 847 participants were included.

- The primary outcomes were depression severity, efficacy of duration/timing of BLT for depressive symptoms, and efficacy of different light color/color temperatures for depressive symptoms.

\section{Outcomes}

- As assessed by the Hamilton Depression Rating Scale (HAM-D); Inventory of Depressive Symptomatology, Clinician Rating; or the Structured Interview Guide for the HAM-D, depression severity significantly decreased $(P<.05)$ with BLT intensity $\geq 5,000$ lux when compared with placebo.

- Subgroup analyses suggested that BLT can improve depression severity with or without adjuvant therapy. Duration of $<10$ hours and $>10$ hours with morning light vs morning plus evening light therapy all produced a significant decrease in depressive symptoms $(P<.05)$.

-White light therapy also significantly decreased depression severity $(P<.05)$.

\section{Clinical Point \\ One meta-analysis found that BLT at various durations, timings, colors, and color temperatures reduced depression severity}




\section{Clinical Point}

\section{A review of 7 RCTs \\ found a small to moderate but significant effect of BLT in reducing depressive} symptoms
Color temperatures $>4,500 \mathrm{~K}$ and $<4,500 \mathrm{~K}$ both significantly decreased depression severity $(P<.05)$.

\section{Conclusion}

- BLT (at various durations, timings, colors, and color temperatures) can reduce depression severity.

- This analysis only included studies that showed short-term improvements in depressive symptoms, which brings into question the long-term utility of BLT.

2. Lam RW, Teng MY, Jung YE, et al. Light therapy for patients with bipolar depression: systematic review and meta-analysis of randomized controlled trials. Can J Psychiatry. 2020;65(5):290-300.

Lam et $\mathrm{al}^{13}$ examined the role of BLT for patients with bipolar depression in a systematic review and meta-analysis.

\section{Study design}

- Investigators conducted a systematic review of RCTs of BLT for patients with bipolar depression. Articles were obtained from Web of Science, Embase, MEDLINE, PsycInfo, and Clinicaltrials.gov using the search terms "light therapy," "phototherapy," "light treatment," and "bipolar."

- Inclusion criteria required patients diagnosed with bipolar disorder currently experiencing a depressive episode, a clinician-rated measure of depressive symptomatology, a specific light intervention, and a randomized trial design with a control.

- A total of 7 RCTs with 259 participants were reviewed. The primary outcome was improvement in depressive symptoms based on the 17-item HAM-D.

\section{Outcomes}

- BLT was associated with a significant improvement in clinician-rated depressive symptoms $(P=.03)$.

- Data for clinical response obtained from 6 trials showed a significant difference favoring BLT vs control $(P=.024)$.
Data for remission obtained from 5 trials showed no significant difference between BLT and control $(P=.09)$.

- Compared with control, BLT was not associated with an increased risk of affective switches $(P=.67)$.

\section{Conclusion}

- This study suggests a small to moderate but significant effect of BLT in reducing depressive symptoms.

- Study limitations included inconsistent light parameters, short follow-up time, small sample sizes, and the possibility that control conditions had treatment effects (eg, dim light as control vs no light).

3. Hirakawa $H$, Terao $T$, Muronaga $M$, et al. Adjunctive bright light therapy for treating bipolar depression: a systematic review and meta-analysis of randomized controlled trials. Brain Behav. 2020;10(12):ee01876. doi. org/10.1002/brb3.1876

Hirakawa et $\mathrm{al}^{14}$ assessed the role of adjunctive BLT for treating bipolar depression. Previous meta-analyses focused on casecontrol studies that assessed the effects of BLT and sleep deprivation therapy on depressive symptoms, but this meta-analysis reviewed RCTs that did not include sleep deprivation therapy.

\section{Study design}

- Two authors searched Embase, MEDLINE, Scopus, Cochrane Central Register of Controlled Trials (CENTRAL), CINAHL, and Clinicaltrials.gov from inception to September 2019 using the terms "light therapy," "phototherapy," and "bipolar disorder."

- Inclusion criteria called for RCTs, participants age $\geq 18$, a diagnosis of bipolar disorder according to standard diagnostic criteria, evaluation by a standardized scale (HAM-D, Montgomery-Åsberg Depression Rating Scale [MADRS], Structured Interview Guide for the Hamilton Depression Rating Scale with Atypical Depression Supplement [SIGH-ADS]), 
and light therapy as the experimental group intervention.

- The main outcomes were response rate (defined as $\geq 50 \%$ reduction in depression severity based on a standardized scale) and remission rate (defined as a reduction to 7 points on HAM-D, reduction to 9 points on MADRS, and score $<8$ on SIGH-ADS).

- Four RCTs with a total of 190 participants with bipolar depression were evaluated.

\section{Outcomes}

- BLT had a significant effect on response rate $(P=.002)$.

- There was no significant effect of BLT on remission rates $(P=.34)$.

- No studies reported serious adverse effects. Minor effects included headache (14.9\% for BLT vs $12.5 \%$ for control), irritability ( $4.26 \%$ for BLT vs $2.08 \%$ for control), and sleep disturbance $(2.13 \%$ for BLT vs $2.08 \%$ for control). The manic switch rate was $1.1 \%$ in BLT vs $1.2 \%$ in control.

\section{Conclusion}

- BLT is effective in reducing depressive symptoms in bipolar disorder, but does not affect remission rates.

- This meta-analysis was based on a small number of RCTs, and light therapy parameters were inconsistent across the studies. Furthermore, most patients were also being treated with mood-stabilizing or antidepressant medications.

- It is unclear if BLT is effective as monotherapy, rather than as adjunctive therapy.

\section{D'Agostino A, Ferrara P, Terzoni S, et al.} Efficacy of triple chronotherapy in unipolar and bipolar depression: a systematic review of available evidence. J Affect Disord. 2020;276:297-304.

Triple chronotherapy is the combination of total sleep deprivation, sleep phase advance, and BLT. D'Agostino et $\mathrm{al}^{15}$ reviewed all available evidence on the efficacy of triple chronotherapy interventions in treating symptoms of major depressive disorder (MDD) and bipolar depression.

\section{Study design}

- Researchers conducted a systematic search on PubMed, Scopus, and Embase from inception to December 2019 using the terms "depression," "sleep deprivation," "chronotherapy," and related words.

- The review included studies of all execution modalities, sequences of interventions, and types of control groups (eg, active control vs placebo). The population included participants of any age with MDD or bipolar depression.

- Two authors independently screened studies. Six articles published between 2009 and 2019 with a total of 190 patients were included.

\section{Outcomes}

- All studies reported improvement in HAM-D scores at the end of treatment with triple chronotherapy, with response rates ranging from $50 \%$ to $84 \%$.

- Most studies had a short follow-up period (up to 3 weeks). In these studies, response rates ranged from $58.3 \%$ to $61.5 \%$. One study that had a 7-week follow-up also reported a statistically significant response rate in favor of triple chronotherapy.

- Remission rates, defined by different cut-offs depending on which version of the HAM-D was used, were evaluated in 5 studies. These rates ranged from $33.3 \%$ to $77 \%$.

- Two studies that used the Columbia Suicide Severity Rating Scale to assess the effect of triple chronotherapy on suicide risk reported a significant improvement in scores.

\section{Conclusion}

- Triple chronotherapy may be an effective and safe adjunctive treatment for depression. Some studies suggest that it also may play a role in remission from depression and reducing suicide risk.

\section{Dallaspezia S, Benedetti F. Antidepressant light therapy for bipolar patients: a meta- analyses. J Affect Disord. 2020;274:943-948.}

Clinical Point

In an analysis of 4 RCTs with 190 participants, BLT significantly improved response rates, but not remission rates 


\section{Clinical Point}

Triple chronotherapy, which includes BLT, may be effective for the treatment of patients with MDD or bipolar depression
In a meta-analysis, Dallaspezia and Benedetti ${ }^{16}$ evaluated 11 studies to assess the role of BLT for treating depressive symptoms in patients with bipolar disorder.

\section{Study design}

- Researchers searched literature published on PubMed with the terms "mood disorder," "depression," and "light therapy."

- Eleven studies with a total of 195 participants were included. Five studies were RCTs.

- The primary outcome was severity of depression based on scores on the HAM-D, Beck Depression Inventory, or SIGH-ADS. Secondary outcomes were light intensity (measured in lux) and duration of treatment.

\section{Outcomes}

- Analysis of all 11 studies revealed a positive effect of BLT on depressive symptoms $(P<.001)$.

- Analysis of just the 5 RCTs found a significant effect of BLT on depressive symptoms $(P<.001)$.

- The switch rate due to BLT was lower than rates for patients being treated with antidepressant monotherapy (15\% to $40 \%$ ) or placebo $(4.2 \%)$.

- Duration of treatment influenced treatment outcomes $(P=.05)$; a longer duration resulted in the highest clinical effect. However, regardless of duration, BLT showed higher antidepressant effects than placebo.

- Higher light intensity was also found to show greater efficacy.

\section{Conclusion}

- BLT is an effective adjunctive treatment for bipolar depression.

- Higher light intensity and longer duration of BLT may result in greater antidepressant effects, although the optimum duration and intensity are unknown.

- A significant limitation of this study was that the studies reviewed had high heterogeneity, and only a few were RCTs.
6. Takeshima $M$, Utsumi $T$, Aoki $Y$, et al. Efficacy and safety of bright light therapy for manic and depressive symptoms in patients with bipolar disorder: a systematic review and meta-analysis. Psychiatry Clin Neurosci. 2020;74(4):247-256.

Takeshima et $\mathrm{al}^{17}$ conducted a systematic review and meta-analysis to evaluate the efficacy and safety of BLT for manic and depressive symptoms in patients with bipolar disorder. They also evaluated if BLT could prevent recurrent mood episodes in patients with bipolar disorder.

\section{Study design}

- Researchers searched for studies of BLT for bipolar disorder in MEDLINE, CENTRAL, Embase, PsychInfo, and Clincialtrials.gov using the terms "bipolar disorder," "phototherapy," and "randomized controlled trial."

- Two groups of 2 authors independently screened titles and abstracts for the following inclusion criteria: RCTs, $80 \%$ of patients diagnosed clinically with bipolar disorder, any type of light therapy, and control groups that included sham treatment or no light. Three groups of 2 authors then evaluated the quality of the studies and risk of bias.

- Six studies with a total of 280 participants were included.

- Primary outcome measures included rates of remission from depressive or manic episodes, rates of relapse from euthymic states, and changes in score on depression or mania rating scales.

\section{Outcomes}

- No significant differences were found between BLT and placebo for rates of remission from depressive episodes $(P=.42)$, rates of manic switching $(P=.26)$, or depressive symptom scores $(P=.30)$.

- Sensitivity analysis for 3 studies with low overall indirectness revealed that BLT did have a significant antidepressant effect $(P=.006)$. 


\section{Clinical Point}

A review of 6 studies found no significant differences between BLT and placebo on several measures of bipolar depression continued from page 44

- The most commonly reported adverse effects of BLT were headache (4.7\%) and sleep disturbance $(1.4 \%)$.

\section{Conclusion}

- This meta-analysis suggests that BLT does not have a significant antidepressant effect. However, a sensitivity analysis of studies with low overall indirectness showed that BLT does have a significant antidepressant effect.

- This review was based on a small number of RCTs that had inconsistent placebos (dim light, negative ion, no light, etc.) and varying parameters of BLT (light intensity, exposure duration, color of light), which may have contributed to the inconsistent results.

\section{References}

1. Diagnostic and statistical manual of mental disorders, 5th ed. American Psychiatric Association; 2013.

2. Judd LL, Akiskal HS, Schettler PJ, et al. The long-term natural history of the weekly symptomatic status of bipolar I disorder. Arch Gen Psychiatry. 2002;59(6):530-537.

3. Judd LL, Akiskal HS, Schettler PJ, et al. A prospective investigation of the natural history of the long-term weekly symptomatic status of bipolar II disorder. Arch Gen Psychiatry. 2003;60(3):261-269.

4. Rihmer Z. S34.02 - Prediction and prevention of suicide in bipolar disorders. European Psychiatry. 2008;23(S2):S45-S45.

5. Simon GE, Bauer MS, Ludman EJ, et al. Mood symptoms, functional impairment, and disability in people with bipolar disorder: specific effects of mania and depression. J Clin Psychiatry. 2007;68(8):1237-1245.
6. Dome P, Rihmer Z, Gonda X. Suicide risk in bipolar disorder: a brief review. Medicina (Kaunas). 2019;55(8):403.

7. Sachs GS, Nierenberg AA, Calabrese JR, et al. Effectiveness of adjunctive antidepressant treatment for bipolar depression. N Engl J Med. 2007;356(17):1711-1722.

8. Post RM, Altshuler LL, Leverich GS, et al. Mood switch in bipolar depression: comparison of adjunctive venlafaxine, bupropion, and sertraline. Br J Psychiatry. 2006;189:124-131.

9. Shah N, Grover S, Rao GP. Clinical practice guidelines for management of bipolar disorder. Indian J Psychiatry. 2017;59(Suppl 1):S51-S66.

10. Penders TM, Stanciu CN, Schoemann AM, et al. Bright light therapy as augmentation of pharmacotherapy for treatment of depression: a systematic review and meta-analysis. Prim Care Companion CNS Disord. 2016;18(5). doi: 10.4088/ PCC.15r01906.

11. Terman M, Amira L, Terman JS, et al. Predictors of response and nonresponse to light treatment for winter depression. Am J Psychiatry. 1996;153(11):1423-1429.

12. Wang $\mathrm{S}$, Zhang $\mathrm{Z}$, Yao L, et al. Bright light therapy in treatment of patients with bipolar disorder: a systematic review and meta-analysis. PLoS ONE. 2020;15(5):e0232798. doi: 10.1371/journal.pone.0232798

13. Lam RW, Teng MY, Jung YE, et al. Light therapy for patients with bipolar depression: systematic review and metaanalysis of randomized controlled trials. Can J Psychiatry. 2020;65(5):290-300

14. Hirakawa H, Terao T, Muronaga M, et al. Adjunctive bright light therapy for treating bipolar depression: a systematic review and meta-analysis of randomized controlled trials. Brain Behav. 2020;10(12):ee01876. doi.org/10.1002/ brb3.1876

15. D'Agostino A, Ferrara P, Terzoni S, et al. Efficacy of triple chronotherapy in unipolar and bipolar depression: a systematic review of available evidence. J Affect Disord. 2020;276:297-304.

16. Dallaspezia S, Benedetti F. Antidepressant light therapy for bipolar patients: a meta-analyses. J Affect Disord. 2020 274:943-948.

17. Takeshima M, Utsumi T, Aoki Y, et al. Efficacy and safety of bright light therapy for manic and depressive symptoms in patients with bipolar disorder: a systematic review and meta-analysis. Psychiatry Clin Neurosci. 2020;74(4): 247-256. 